\title{
Control of bleeding in surgical procedures: critical appraisal of HEMOPATCH (Sealing Hemostat)
}

This article was published in the following Dove Press journal:

Medical Devices: Evidence and Research

22 December 2015

Number of times this article has been viewed

\section{Kevin Michael Lewis' \\ Carl Erik Kuntze ${ }^{2}$ \\ Heinz Gulle ${ }^{3}$}

'Preclinical Safety and Efficacy, Baxter Healthcare Corporation, Deerfield, IL, USA; ${ }^{2}$ Medical Affairs, Baxter Healthcare SA, Zurich, Switzerland; ${ }^{3}$ Surgical Sciences and Engineering, Baxter Medical Products $\mathrm{GmbH}$, Vienna, Austria

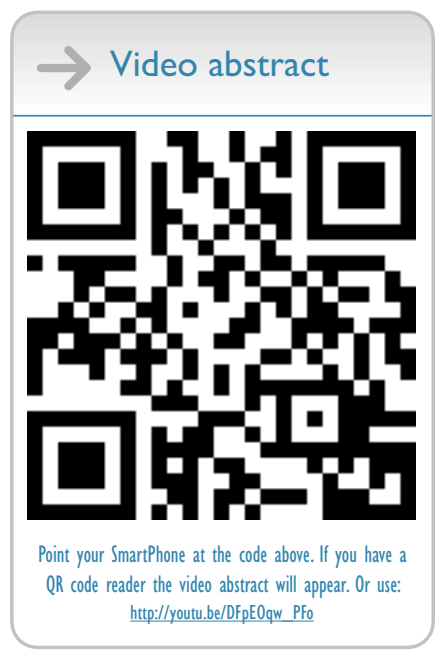

Correspondence: Kevin Michael Lewis Preclinical Safety and Efficacy, Baxter Healthcare Corporation, One Baxter Parkway, Deerfield, IL 600 I5, USA $\mathrm{Tel}+$ I 2242705484

Fax + I $224270547 \mid$

Email kevin_lewis@baxter.com
Abstract: The need for advanced hemostatic agents increases with the complexity of surgical procedures and use of anticoagulation and antiplatelet treatments. HEMOPATCH (Sealing Hemostat) is a novel, advanced hemostatic pad that is composed of a synthetic, protein-reactive monomer and a collagen backing. The active side is covered with a protein-reactive monomer: N-hydroxysuccinimide functionalized polyethylene glycol (NHS-PEG). NHS-PEG rapidly affixes the collagen pad to tissue to promote and maintain hemostasis. The combined action of the NHS-PEG and collagen is demonstrated to have benefit relative to other hemostatic agents in surgery and preclinical surgical models. This paper reviews the published investigations and case reports of the hemostatic efficacy of HEMOPATCH, wherein HEMOPATCH is demonstrated to be an effective, easy-to-use hemostatic agent in open and minimally invasive surgery of patients with thrombin- or platelet-induced coagulopathies.

Keywords: HEMOPATCH, hemostasis, surgical hemostasis, sealing, surgical sealant

\section{Background}

Medical device development has evolved with the complexity of surgical procedures being performed and the increased use of anticoagulation and antiplatelet therapies, wherein hemostatic agents have continually evolved new features and benefits. ${ }^{1}$ Collagen pads were first introduced for hemostasis and wound healing in $1980 .{ }^{2}$ Collagen pads were then coated with fibrinogen and thrombin to improve hemostatic performance and to provide tissue sealing in $1987 .{ }^{3}$ Though these hemostatic pads have been used in surgery successfully for several decades, new requirements drive the development of novel hemostatic pads. Three new hemostatic pads have recently been developed and used in clinical practice, ie, HEMOPATCH,${ }^{4}$ VERISET, ${ }^{5}$ and EVARREST. ${ }^{6}$

These hemostatic pads consist of a sheet-like backing and a self-adhering surface. The various backings include collagen, neutralized oxidized cellulose, or an oxidized cellulose-polyglactin 910 composite; while the active surfaces include fibrinogen and thrombin or a synthetic, protein-reactive monomer. ${ }^{4-6}$ Of the various combinations, collagen provides advantages over the acidic nature of oxidized cellulose; and synthetic, protein-reactive monomers provide advantages over human-derived proteins. This paper provides a critical review of an advanced hemostatic pad composed of collagen and a synthetic, protein-reactive monomer, HEMOPATCH (Sealing Hemostat) (Baxter AG, Vienna, Austria). This paper describes the components, mechanism of action, and approval status of HEMOPATCH; reviews published preclinical and clinical data; and offers areas for future development and study. submit your manuscript | www.dovepress.com

Dovepress

http://dx.doi.org// 0.2147/MDER.S9059/

Medical Devices: Evidence and Research 2016:9 I-10






\section{HEMOPATCH (Sealing Hemostat)}

HEMOPATCH consists of a specifically formulated collagen matrix, a protein-reactive monomer, and a biocompatible dye (Figure 1). The collagen pad is derived from bovine dermis sourced from countries with negligible bovine spongiform encephalopathy risk according to the classification of the World Organization for Animal Health. ${ }^{7}$ The collagen pad is optimized to be soft, thin, pliable, and of high liquid absorption capacity. This collagen pad provides HEMOPATCH with properties necessary for easy tissue conformance and simple use in open and minimally invasive surgery. ${ }^{8}$

The active side of the collagen pad is coated with a thin layer of a four-armed cross-linking agent, pentaerythritol poly(ethylene glycol) ether tetra-succinimidyl glutarate N-hydroxysuccinimide functionalized polyethylene glycol (NHS-PEG), which is a rapid protein-reactive monomer. Due to the well-known biocompatibility, NHS-PEG is also a component of various medical devices such as vascular, dural, and lung sealants. ${ }^{9}$ The nonactive side of the collagen pad is marked with blue squares for easy differentiation. The blue squares are spaced at a distance of $1 \mathrm{~cm}$ from each other to facilitate measuring and cutting. The blue squares are a low concentration of Brilliant Blue (FD\&C Blue No 1), a clinically established and well-tolerated dye also used as a food additive ${ }^{10,11}$ and as a colorant in drug formulations and surgical sealants. ${ }^{12}$

\section{Mechanism of action}

The performance of HEMOPATCH is based on a unique dual mechanism of action that creates rapid and lasting hemostasis



Figure I HEMOPATCH (Sealing Hemostat) is a next-generation hemostatic pad. Notes: HEMOPATCH is composed of a bovine collagen backing and pentaerythritol polyethylene glycol ether tetra-succinimidyl glutarate (NHS-PEG) monomer that seals and induces hemostasis of bleeding tissue. The blue squares in the HEMOPATCH indicate the nonactive side and are spaced $\mathrm{I} \mathrm{cm}$ apart.

Abbreviation: NHS-PEG, N-hydroxysuccinimide functionalized polyethylene glycol. by sealing the bleeding surface and promoting hemostasis. The rapid adherence and tissue sealing is produced by the NHS-PEG monomers and the hemostatic action is produced by the collagen backing.

\section{Adherence and sealing}

The first mechanism is rapid adherence upon application to tissue due to the electrophilic cross-linking action of NHSPEG. Depending on the $\mathrm{pH}$, the NHS esters of NHS-PEG readily bind to proteins, specifically the $\alpha$-amino group on the $\mathrm{N}$-terminus of proteins and the $\varepsilon$-amino group of lysine. ${ }^{13}$ By doing so, amine bonds form. Therefore, when the NHS-PEG of HEMOPATCH is activated and hydrolyzed upon contact with blood or other body fluids, the resulting molecular components covalently bind with proteins in the blood, or other body fluids, and the tissue surface on which HEMOPATCH is applied (Figure 2). The amine bonds function to affix HEMOPATCH to tissue.

In addition, cross-linking NHS-PEG and proteins, such as human serum albumin, form a hydrogel, which effectively adheres and seals tissue surfaces. ${ }^{14}$ Due to the electrophilic cross-linking, adherence is not limited to bleeding tissue, but is limited to the presence of a proteinaceous fluid. In vitro experiments have shown that sealing of and adherence to a tissue substrate will occur in the presence of other physiological fluids containing proteins. ${ }^{15}$ The need for the presence of a proteinaceous fluid precludes prophylactic use of HEMOPATCH on dry tissue surfaces as protein-containing fluids are required to form the amide bonds and hydrogel. The rapid formation of the amide bonds and hydrogel also require a new piece of HEMOPATCH to be used when it does not immediately adhere.

\section{Hemostasis}

The second mechanism involves collagen, which has several important functions. Collagen is well known to participate in the intrinsic activation of the blood coagulation process and in activating platelets. ${ }^{16}$ Platelets adhere to collagen via the glycoprotein receptor Ib/IX/V complex on the platelet surface. ${ }^{17}$ In doing so, collagen induces lipid molecules to undergo a transbilayer flip in the platelet membrane, which exposes procoagulant phospholipids on the platelet surface leading to the generation of thrombin and further platelet activation. ${ }^{18}$ The additionally generated thrombin cleaves circulating fibrinogen to form fibrin, which stabilizes the forming platelet plug. ${ }^{19}$ In addition to inducing platelet plug formation, collagen is a vehicle to deliver NHS-PEG to the bleeding surface. 


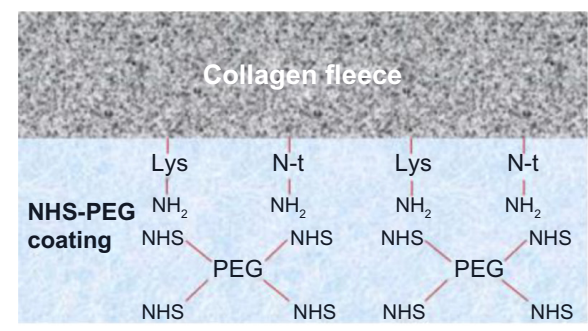

1. Upon application, body fluids dissolve NHS-PEG coating

2. Four-arm NHS-PEG reacts with $\alpha-\mathrm{NH}_{2}$ groups on $\mathrm{N}$-t of proteins and with $\varepsilon-\mathrm{NH}_{2}$ groups on Lys residues

3. Covalent amide bonds are formed, cross-linking PEG and proteins on tissue/blood and collagen

4. NHS molecules are released during the reaction

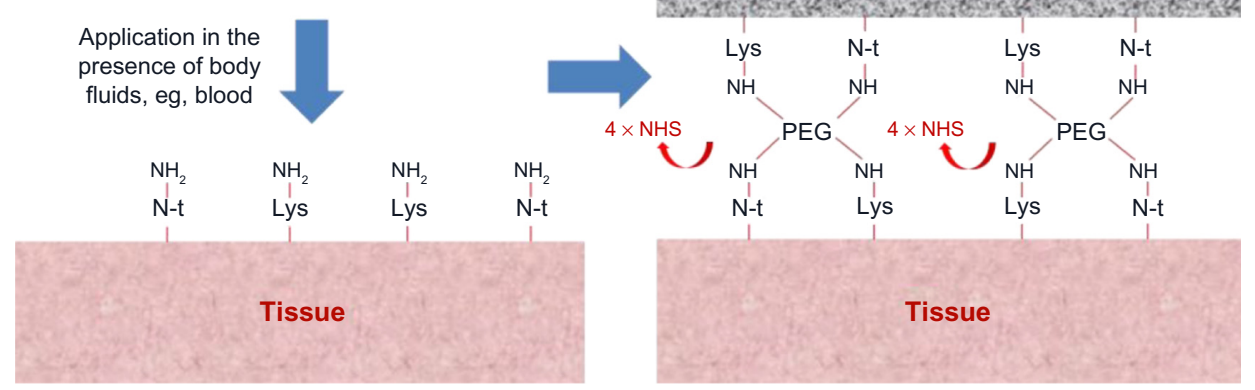

Figure 2 HEMOPATCH (Sealing Hemostat) binds to tissue through covalent amide bonds between PEG and tissue proteins and collagen. Abbreviations: Lys, lysine; N-t, N-terminus; NHS-PEG, N-hydroxylsuccinimide functionalized polyethylene glycol.

On its own, NHS-PEG solutions are quickly washed away by blood or other leaking body fluids. As a result, bonding to tissue surface proteins is very difficult. Many flowable PEG-based surgical sealants require a dry application surface, application after achieving hemostasis, or application to tissues with minimum leakage of body fluids. Physical attachment of dry NHS-PEG to a collagen pad provides a novel vehicle to overcome this limitation. Furthermore, due to the open pore structure of the collagen, excess fluids are readily absorbed and direct contact of NHS-PEG to tissue surface is achieved (Figure 3). The open pore structure is favored for

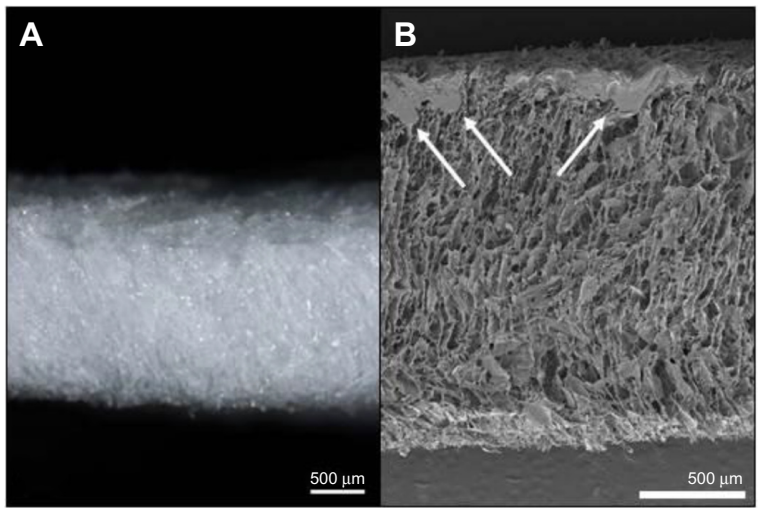

Figure 3 HEMOPATCH (Sealing Hemostat) collagen has an open pore structure that absorbs excess tissue fluid and delivers NHS-PEG to the tissue surface. Notes: The active surface is positioned upward and the open pore structure is seen throughout the collagen cross section using (A) stereoscopy imaging and (B) SEM imaging. Pockets of NHS-PEG powder are seen on the active surface in the SEM image (arrows).

Abbreviations: NHS-PEG, N-hydroxylsuccinimide functionalized polyethylene glycol; SEM, scanning electron microscope. hemostatic pads as it is believed to reduce adhesive failures secondary to continued blood loss. ${ }^{20}$ Finally, blood penetrating into the pad will encounter a dense network of collagen fibers, which provide a structural backbone for platelet plug and fibrin clot formation.

\section{Other properties}

While collagen can swell secondarily to absorbing tissue fluid, PEG-based sealants are known to swell more than 300 times their volume. ${ }^{21}$ The swell property is caused by the composition and hydrophilic nature of the PEG-polymer, which draws in tissue fluid, saline and water. PEG-based sealants are composed of two reaction partners in fluid states that rapidly form a hydrogel with each other upon application. Initial swelling of these hydrogels takes place when further fluid is absorbed and additional swelling occurs during degradation of the PEG-polymer due to hydrolysis of the chemical bonds. Unlike these sealants, HEMOPATCH consists of a single reactive $\mathrm{PEG}$ and a solid collagen matrix.

Upon application, the NHS-PEG quickly dissolves and, due to the open pore structure and application pressure, penetrates into the collagen fleece. Hence, swelling is restricted by the physical properties of the collagen fleece. An in vitro investigation of HEMOPATCH measured the initial noncompressed thickness to be $2.0 \mathrm{~mm}$, while the maximum thickness when submerged in citrated human plasma for 24 hours is $2.8 \mathrm{~mm}$, an increase of $40 \% .{ }^{15}$ However when pressure is applied to mimic application, the mean thickness at 24 hours was $2.1 \pm 0.2 \mathrm{~mm}$ (mean \pm standard deviation [SD], $\mathrm{N}=10$ ), 
suggesting a limited swell. This minimal capacity to swell limits the secondary complications of "mass effect." It should, however, be noted that swell can still occur and that judicious amounts should be used in or around nervous or expandable tissue.

\section{Global registration of HEMOPATCH}

The novel dual mechanism of action creates an effective hemostatic agent and tissue sealant, which at the time of this publication is approved in more than 25 countries worldwide. HEMOPATCH is approved as a hemostatic device for surgical procedures when control of bleeding by pressure, ligature, or conventional procedures is either ineffective or impractical. As approved, HEMOPATCH is applied dry with the active surface to the tissue and approximated using dry gauze (Figure 4).

\section{Preclinical studies}

The efficacy of HEMOPATCH was first investigated in two different animal models of hepatic surgery. ${ }^{4}$ In this investigation, HEMOPATCH was compared with a fibrinogen-thrombin-coated collagen pad, TACHOSIL (Takeda Pharmaceutical Company, Osaka-shi, Japan). The authors used a nonheparinized rabbit hepatic segmentectomy to mimic the clinical procedure and a heparinized porcine hepatic abrasion model to mimic a capsular tear, adhesiolysis, or trauma secondary to manipulation.

In the rabbit hepatic segmentectomy treatment comparison, intraoperative time to hemostasis, hemostatic success, and hematoma formation were assessed. Postoperatively, hematoma formation was also assessed at 24 hours. The hepatic segmentectomy bleed is characterized as a mild, diffuse bleed. In this study, HEMOPATCH provided faster time to hemostasis, greater hemostatic success, and lower incidence of hematoma formation as compared with
TACHOSIL. Most notably, the probability of hemostasis immediately after application was 100\% (95\% confidence interval [CI]: $72.3 \%-100 \%, \mathrm{~N}=12$ ) in the HEMOPATCH group ( 2 minutes) and 80.0\% (95\% CI: 49.0\%-94.3\%, N=12) in the TACHOSIL group (3 minutes) (Figure 5). Similarly, HEMOPATCH provided a lower incidence of hematoma formation at 24 hours as compared with TACHOSIL, 20.0\% versus $66.7 \%$, respectively.

In the porcine hepatic abrasion treatment comparison, bleeding rates, hemostatic success, and hematoma formation were assessed. The animals in this study were heparinized to 1.5-2 times their baseline activated clotting time (ACT) to mimic the coagulopathy secondary to acute and chronic liver disease. Under these conditions, the bleed is characterized as a mild, localized bleed. In this study, HEMOPATCH provided superior hemostatic success and statistically significant less blood loss relative to TACHOSIL (Figure 6). At 3 minutes after application, the odds of hemostatic success of HEMOPATCH to TACHOSIL was 24.8 (95\% CI: 8.86-69.2, $\mathrm{N}=42$ per group). Similarly, HEMOPATCH provided a lower incidence of hematoma formation intraoperatively than did TACHOSIL (14.3\% vs $42.9 \%$ ).

These initial investigations demonstrated the superiority of HEMOPATCH to TACHOSIL in two animal models of hepatic surgery. Based on the odds ratio of hemostatic success, HEMOPATCH was 24 times more likely to stop clinically relevant mild bleeds relative to TACHOSIL. The investigations mimicked clinically relevant bleeds (ie, wide, raw bleeding surfaces, and capsular oozing) and patient conditions (ie, coagulopathy). The authors state that the efficacy difference between the hemostatic agents is due to the fast adherence of the protein-reactive NHS-PEG compared with the slower enzymatic conversion of fibrinogen to fibrin. As a result, it is presumed that this slower adherence rate led to greater hematoma formation beneath TACHOSIL resulting in greater hemostatic failure.
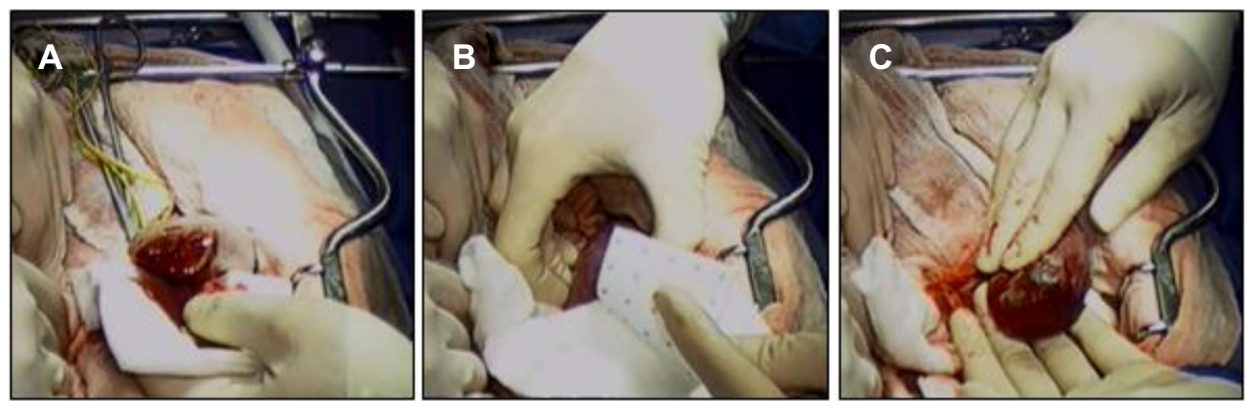

Figure 4 HEMOPATCH (Sealing Hemostat) is applied to a partial nephrectomy in a swine.

Notes: The partial nephrectomy is $\sim \mathrm{cm}$ deep. The bleeding tissue is identified (A), orientation of HEMOPATCH is confirmed (B), and complete hemostasis is achieved following application (C). 

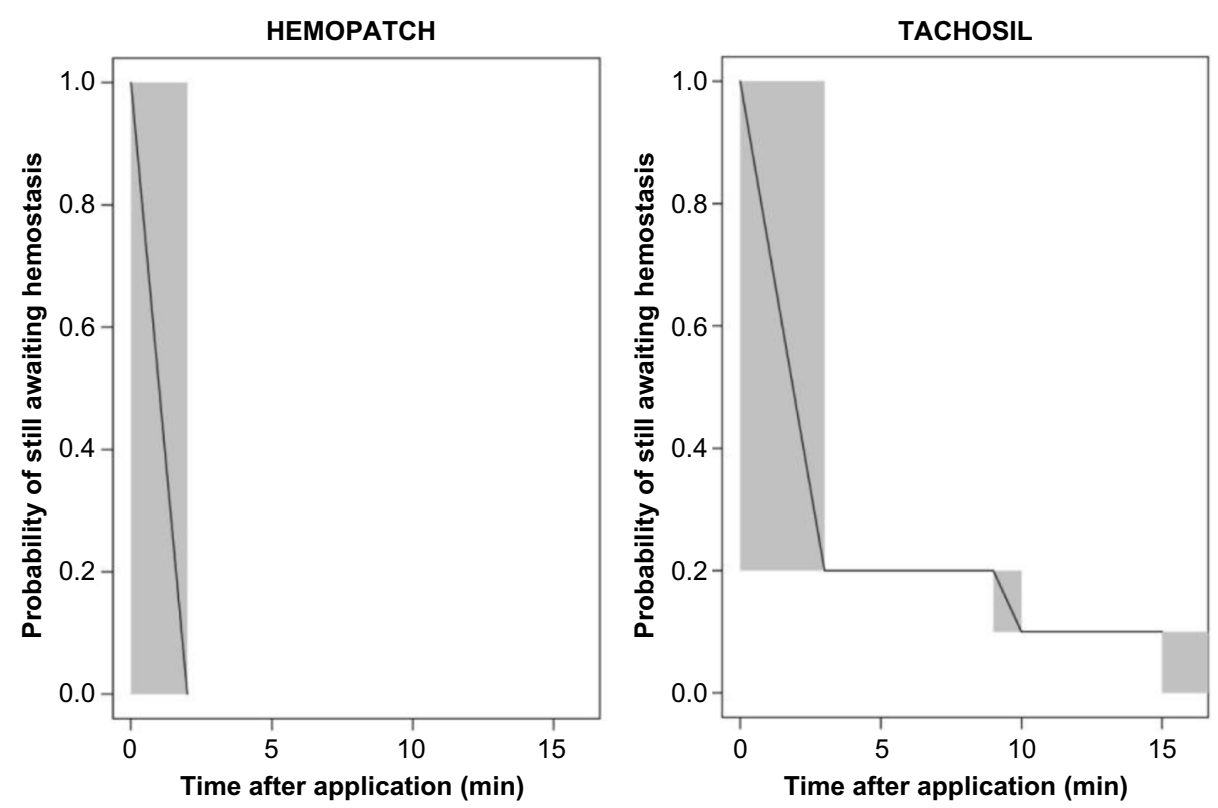

Figure 5 HEMOPATCH provided 100\% hemostasis immediately after application, while TACHOSIL provided $80 \%$ hemostasis.

Notes: Kaplan-Meier plot for interval censored time of hemostasis, where the time interval during which hemostasis occurred is shaded. Adapted from Lewis KM, McKee J, Schiviz A, Bauer A, Wolfsegger M, Goppelt A. Randomized, controlled comparison of advanced hemostatic pads in hepatic surgical models. ISRN Surg. 20I4;20I4:930803. ${ }^{4}$

The efficacy of HEMOPATCH was then investigated in both a vascular injury and hepatic lesion animal model. ${ }^{22}$ In this investigation, HEMOPATCH was compared with oxidized cellulose, SURGICEL (Ethicon Inc., Somerville, NJ, USA). The authors used a heparinized rabbit vascular injury model to mimic bleeding from needle holes following a vascular reconstruction with graft placement or a vascular anastomosis following organ transplant, and a heparinized porcine hepatic square lesion model to mimic a capsular tear, adhesiolysis, or trauma secondary to manipulation.
In the rabbit vascular model, hemostatic effectiveness and probability of hemostatic success were assessed intraoperatively. The vascular bleed is characterized as an arterial spurt and had a median bleed rate of $14.5 \mathrm{~mL} / \mathrm{min}$. The animals in this study were heparinized to a clinically relevant ACT of 250 seconds, which is used for peripheral graft placement. ${ }^{23}$ In this study, HEMOPATCH provided superior efficacy relative to SURGICEL based on an odds of hemostatic success of 85.3 (95\% CI: $25.8-282, \mathrm{~N}=40$ per group) at 2 minutes after application. In this context,
A

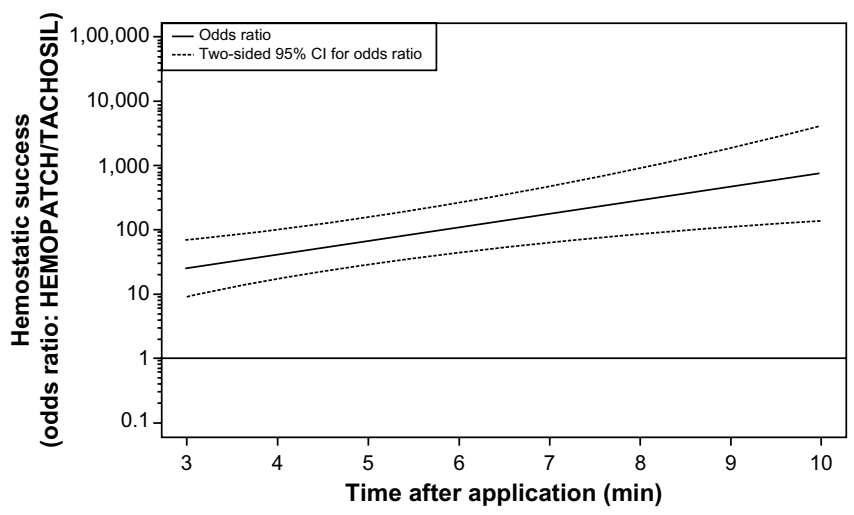

B

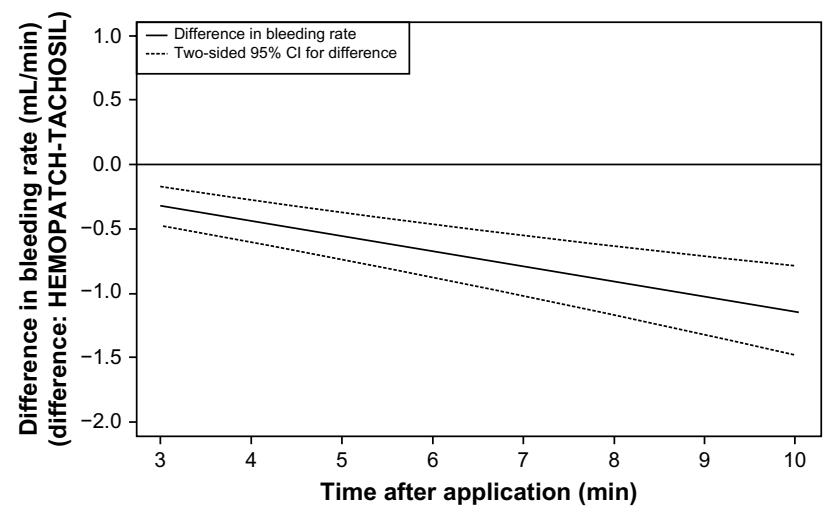

Figure 6 HEMOPATCH provides superior hemostatic performance and significantly less blood loss than TACHOSIL.

Notes: Odds ratio of hemostatic success and $95 \% \mathrm{Cl}$, where the odds ratio of hemostatic success of HEMOPATCH is divided by that of TACHOSIL (A). HEMOPATCH had a statistically significant, at the $5 \%$ level, superior hemostatic success over time as the lower $95 \% \mathrm{Cl}$ is $>$ I. Difference in rate of blood loss from lesions treated with HEMOPATCH and those treated with TACHOSIL, where the bleeding rate of TACHOSIL is subtracted from that of HEMOPATCH (B). HEMOPATCH had a statistically significant, at the $5 \%$ level, less bleeding rate over time as the upper $95 \% \mathrm{Cl}$ is <0. Adapted from Lewis KM, McKee J, Schiviz A, Bauer A, Wolfsegger M, Goppelt A. Randomized, controlled comparison of advanced hemostatic pads in hepatic surgical models. ISRN Surg. 20I4;20I4:930803. ${ }^{4}$

Abbreviation: $\mathrm{Cl}$, confidence interval. 
the calculated probability of success of HEMOPATCH was $80.1 \%$ at 2 minutes, which increased to $100 \%$ at 10 minutes after application, while that of SURGICEL was $4.7 \%$ and $99.6 \%$, relatively.

In the porcine hepatic square model, hemostatic success was assessed. The model is widely accepted in literature for this type of comparison. ${ }^{24}$ In this investigation, the model is characterized as being normotensive, that is, mean blood pressure of $78 \pm 13 \mathrm{mmHg}$ (mean $\pm \mathrm{SD}, \mathrm{N}=80$ ), with a systolic of $102 \pm 13 \mathrm{mmHg}$ and diastolic of $63 \pm 13 \mathrm{mmHg}$ throughout the investigation. The hypocoagulopathy induced was between $1.4 \times$ and $3.6 \times$ baseline ACT throughout the investigation. Under these conditions, HEMOPATCH provided superior hemostatic success relative to SURGICEL up to 8 minutes after application.

These follow-up investigations demonstrated the superiority of HEMOPATCH to SURGICEL in a vascular and hepatic animal model. In this model, based on the odds ratio of hemostatic success, HEMOPATCH was 85 times more likely to stop a clinically relevant moderate bleed relative to SURGICEL. The authors stated that the superior efficacy of HEMOPATCH relative to SURGICEL was due to the unique dual mechanism of action of HEMOPATCH. The efficacy of oxidized cellulose was limited by its hemostyptic action generated by its fluid absorption and low $\mathrm{pH}$ to stop this moderate-level bleeding. ${ }^{25}$

These four animal models demonstrate how the proteinreactive monomer and procoagulant collagen backing of HEMOPATCH combine to be a unique dual mechanism of action. The studies, however, only investigate the efficacy of HEMOPATCH in mild and moderate bleeds. The studies do not investigate its efficacy to treat severe bleeds and in tissue sealing applications. The majority of bleeding faced in surgery is mild and moderate, yet understanding the maximum treatable level of bleeding is important to understand the hemostatic utility. Therefore, an additional preclinical study investigated the efficacy of HEMOPATCH in a severe bleed and in tissue sealing applications.

The efficacy of HEMOPATCH to treat a severe bleed was investigated using a nonanatomical pulmonary segmentectomy model of lung surgery. ${ }^{15}$ The pulmonary segmentectomy is characterized as a severe arterial bleed with a bleeding rate of $17.7 \pm 8.6 \mathrm{~mL} / \mathrm{min}$ (mean $\pm \mathrm{SD}, \mathrm{N}=36$ ) from an area of $5.4 \pm 3.3 \mathrm{~cm}^{2}$. The model was performed under normotensive (ie, systolic blood pressure: $97 \pm 9 \mathrm{mmHg}$, diastolic: $62 \pm 10 \mathrm{mmHg}$ ) and hypocoagulopathic (ie, ACT of $179 \pm 43$ seconds) conditions, while the lung was actively ventilated at $15 \pm 4$ breaths per minute with an airway pressure of $19 \pm 2 \mathrm{~cm} \mathrm{H}_{2} \mathrm{O}$. HEMOPATCH reduced and maintained the rate of bleeding to be negligible 3 minutes after application $(0.2 \pm 0.47 \mathrm{~mL} / \mathrm{min})$. Hematoma development was not noted between the treatment and lung tissue for any treated lesion.

In addition to the reduction and maintenance of bleeding, the rate of intraoperative air leakage was $11 \%$ in the 36 applications. As the authors cited, this is very low relative to clinically reported incidence of intraoperative air leaks. The rate of intraoperative air leaks following standard of care treatment was $71 \%-90 \%$ and for TACHOSIL treatment was $66 \%-70 \%{ }^{26,27}$ While this study demonstrates favorable intraoperative performance, the long-term performance to reduce persistent air leaks was not assessed.

In this same publication, the authors investigated the efficacy of HEMOPATCH to adhere to tissue substrates with various body fluids. By using an in vitro test system, HEMOPATCH provided burst pressures sufficient to withstand normal tissue pressures of fluid-filled cavities (Table 1). The authors noted that HEMOPATCH fixation functioned independent of the coagulation cascade and, therefore, led to more consistent performance than other hemostatic agents that depended on the coagulation cascade.

Based on the preclinical data, HEMOPATCH is a versatile hemostatic sealant. HEMOPATCH was 24 times more likely to provide hemostatic success relative to TACHOSIL, a fibrin and thrombin-coated collagen pad, and 85 times more likely than SURGICEL, an oxidized cellulose, to do so. The superior performance of HEMOPATCH relative to these agents supports that the 1) synthetic, protein-reactive polymer provides rapid adherence and 2) the collagen pad is an effective procoagulant that activates platelets and promotes platelet aggregation. While the preclinical studies demonstrate the utility of the hemostatic and sealing effectiveness of HEMOPATCH, preclinical

Table I HEMOPATCH (Sealing Hemostat) can withstand normal pressures of fluid-filled cavities when applied in the presence and absence of blood on a tissue substrate

\begin{tabular}{lll}
\hline Fluid-filled cavity & $\begin{array}{l}\text { Normal pressure } \\
\text { of cavity }(\mathbf{m m H g})\end{array}$ & $\begin{array}{l}\text { Burst pressure of } \\
\text { HEMOPATCH }(\mathbf{m m H g})\end{array}$ \\
\hline Subarachnoid space & $7-15$ & $19.8-38.2$ \\
Gallbladder & $2.1-12.2$ & $19.0-23.6$ \\
Lymphatic system & $-^{\mathrm{a}}$ & $29.9-32.8$ \\
Urinary bladders & $5-25$ & $19.0-36.5$ \\
\hline
\end{tabular}

Notes: a Data not available. Data from Lewis KM, Spazierer D, Slezak P, Baumgartner B, Regenbogen J, Gulle H. Swelling, sealing, and hemostatic ability of a novel biomaterial: a polyethylene glycol-coated collagen pad.J Biomater Appl. 2014;29(5):780-788. ${ }^{15}$ 
studies do not fully replicate clinical conditions and comorbidities.

\section{Clinical studies}

Imkamp et $\mathrm{al}^{28}$ were the first to provide a clinical report regarding the performance of HEMOPATCH in laparoscopic, zero-ischemia partial nephrectomy. The seven-patient case series details the hemostatic effectiveness of HEMOPATCH to treat the bleeding tissue surface following enucleation of renal tumors. In four of the seven patients, no hemostatic undersuture was used. In all cases, HEMOPATCH was rolled and inserted through a $10 \mathrm{~mm}$ laparoscopic port. When deployed from the port, HEMOPATCH was reported to return to its original configuration after being rolled.

Upon application, hemostasis was reached 2-3 minutes after application in all patients. The postoperative period of all patients was uneventful, no significant drainage was observed, and no suspicious findings were observed on ultrasound. In Imkamp et al's opinion based on the size of the tumor resection sites (up to $72 \mathrm{~mm}$ ), HEMOPATCH might be appropriate to safely treat the cut parenchymal surface following partial nephrectomy.

This study was the first to investigate the hemostatic efficacy of HEMOPATCH applied to laparoscopic moderate bleeding, wherein the authors concluded that HEMOPATCH provided advantage to other hemostatic pads, specifically: an ability to restore its original shape and rigidity when dry to aid intracorporeal handling; rapid and tight fixation on to tissue; and rapid hemostasis and sealing of bleeding tissues.

Prestipino et $\mathrm{al}^{29}$ were the first to provide a clinical report regarding the hemostatic and sealing efficacy of HEMOPATCH. The authors described the use of HEMOPATCH in combination with another hemostatic agent to repair a ventricular perforation by a pacemaker lead. The 92-year-old male patient underwent dual mode, dual chamber, and dual sensing permanent pacemaker implantation for sick sinus syndrome and intermittent atrioventricular block 21 days prior to being presented. At the time of surgery to correct the perforation, chest radiographs revealed a protrusion of the tip of the ventricular electrode through the right ventricle and into the left chest wall. The patient underwent emergency surgery without an opportunity to discontinue antiplatelet therapy (Aspirin, $100 \mathrm{mg}$ once daily).

On intraoperative exam, the surgical team discovered that the lead penetrated through the interventricular septum and left ventricle. The lead was removed and the left ventricle was treated and repaired using FLOSEAL (Baxter International Inc., Deerfield, IL, USA) and then with HEMOPATCH. HEMOPATCH was selected in place of sutures due to the thin and fragile ventricular wall. Despite active antiplatelet therapy, HEMOPATCH stopped bleeding and sealed the ventricular wall. The authors concluded that the use of a hemostatic patch, such as HEMOPATCH, is appropriate to treat a perforation and control bleeding.

Fingerhut et $\mathrm{al}^{8}$ have presented the most comprehensive case series regarding the efficacy of HEMOPATCH, which includes 17 cases totaling 32 applications in solid organ, gastrointestinal, biliopancreatic, endocrine, cardiovascular, and urological surgeries. ${ }^{8}$ These surgeries include anatomical and endoscopic approaches. While there are several notable cases within the series, three of the cases discuss the benefit of HEMOPATCH given the patients' status.

In the first case, a 63-year-old obese male patient with hypertension and hepatitis B virus-related liver cirrhosis underwent an open hepatic 6-7 segment sectionectomy to remove a $5 \mathrm{~cm}$ hepatocellular carcinoma. The surgeons used a harmonic scalpel, and 5-0 polypropylene suture and vessel clips to ligate parenchymal vessels. At the end of the procedure, incomplete hemostasis and biliostasis was noted. The surgeons elected and reported successful use of HEMOPATCH to treat the heavily electrocoagulated hepatic parenchyma. The use of HEMOPATCH minimized blood loss to $200 \mathrm{cc}$ and obviated the need for transfusion.

In the second case, a 54-year-old male patient with diabetes and chronic pancreatitis underwent a Whipple procedure. The surgeons encountered difficult dissection of the portal vein, superior mesenteric artery, and vein. The surgeons elected and reported successful use of HEMOPATCH to treat the bleeding despite the hyperproteolytic environment and hypocoagulopathic condition due to local fibrosis and inflammation. The authors also describe two additional laparoscopic pancreatectomy cases, wherein HEMOPATCH was used to treat the parenchymal cut surfaces. In each case, no postoperative bleeding or pancreatic fistula was observed.

In the third case, a 64-year-old female patient being treated with antiplatelet therapy underwent an exploratory laparotomy during which an anterior resection of the rectum, salpingectomy, and partial resection of the uterus was performed to remove an adherent inflammatory pseudotumor from the sigmoid colon. The patient was unable to discontinue her antiplatelet therapy prior to surgery. Following the extensive resection, the surgeons elected to treat ongoing blood loss with HEMOPATCH, instead of using sutures or energy devices. The sutures and energy devices were determined to be potentially damaging to the ureter. In similar cases, the authors reported using HEMOPATCH to manage diffuse bleeding complications to preserve sensitive structures instead of traditional energy devices, including: a bilateral extracapsular 
thyroidectomy and a robot-assisted nerve-sparing radical prostatectomy. In these cases, HEMOPATCH replaced the need for alternate treatment options that have secondary tissue trauma (eg, electrocautery, harmonic scalpel, argon beam).

Throughout the case series, HEMOPATCH successfully stopped bleeding and sealed tissues despite concurrent disease states (eg, diabetes, hepatitis, cancer, etc) and treatments (eg, chemoradiation, heparinization, antiplatelet drugs, etc) and friable tissue. The case series also supports the use of HEMOPATCH in anatomical and endoscopic surgery, wherein it can be easily applied through a $10 \mathrm{~mm}$ port, applied to vertical and inverted structures, and applied to rounded or uneven surfaces.

In the most recent clinical report, Jainandunsing et $\mathrm{al}^{30}$ described a case in which HEMOPATCH was used for hemostasis and sealing. The 64-year-old patient underwent left anterior descending and anterolateral coronary artery grafting, resection of the interventricular septum and aortic and mitral valve replacement while on cardiopulmonary bypass. In addition to the coagulopathies related to cardiopulmonary bypass (ie, heparinization, hypothermia), the patient was suspected to have endocarditis leading to an increased tendency to bleed.

HEMOPATCH was used to treat the dissected myocardial tissue and the anastomotic suture lines. On postoperative transthoracic echocardiography, no pericardial effusion or pseudoaneurysm suggestive of hemostatic failure were observed. The authors concluded that HEMOPATCH is an appropriate therapy to treat bleeding and maintain wound closure without the need for sutures. Moreover, use of HEMOPATCH did not interrupt coronary blood flow in the graft or compromise cardiac function.

As the clinical evidence for the use of HEMOPATCH increases, surgical teams are exploring the use of HEMOPATCH in their surgical practice. ${ }^{31,32}$ The multiple case reports support the use of HEMOPATCH to provide hemostasis and tissue sealing. The broad clinical use of HEMOPATCH confirms the versatility and utility of the hemostatic agent in coagulopathic, diseased patients with injured or diseased tissue having mildto-severe bleeding. The clinical reports, further, demonstrate that HEMOPATCH can be used safely and effectively in open and minimally invasive applications. Therefore, it is anticipated that the clinical case reports will soon be further expanded to larger, prospective randomized studies.

\section{Future developments}

While there are no unfavorable reports of HEMOPATCH, the majority of the literature is using preclinical animal models. These studies used appropriate controls, which provide an understanding of the hemostatic agent's relative performance. In contrast, the clinical case reports are not designed to compare the safety and efficacy of HEMOPATCH to other hemostatic agents. Therefore, a controlled clinical study is required. Such an investigation should include multiple surgical specialties, and open and endoscopic surgical procedures. Beyond a controlled, clinical study to investigate the hemostatic efficacy of HEMOPATCH, other surgical applications can also be investigated in preclinical models. Based on this performance and the mechanism of action, HEMOPATCH may be efficacious as a surgical sealant as well.

HEMOPATCH affixes to tissue when the NHS-PEG dissolves and polymerizes. As NHS-PEG polymerizes, it affixes the collagen pad to the tissue. This interaction is dependent upon the presence of a proteinaceous fluid. As demonstrated in an in vitro study, the fluid can be bile, lymph, or cerebrospinal fluid and blood. ${ }^{15}$ Of the different fluids investigated, HEMOPATCH withstood clinically relevant pressures to maintain normal intracranial pressure when applied in the presence of cerebrospinal fluid. The ability for HEMOPATCH to affix to dura and seal cerebrospinal fluid leaks is an interesting area of future investigation. HEMOPATCH has a favorable, minimal swell profile limiting a potential "mass effect" and is composed of a collagen backing that is similar to dural substitutes.

In the same preclinical study, the investigators studied the efficacy of HEMOPATCH to provide hemostasis of a pulmonary segmentectomy during active ventilation. ${ }^{15}$ During the 10-minute observation period, the treated lesions had a low incidence of insufficient pneumostasis (11\%, 4 of 36). The investigators did not perform a submersion test to study the burst pressure and did not report a control arm; therefore, additional studies to investigate the pneumostatic ability of HEMOPATCH are of interest. Overall, based on the mechanism of action, additional areas of future investigation include the sealing efficacy of other protein-rich organs as well (eg, pancreas, gallbladder, bladder).

\section{Conclusion}

Through numerous preclinical and clinical case studies, HEMOPATCH (Sealing Hemostat) is proving to be an effective hemostatic agent and sealant for use in open and endoscopic surgical procedures. The unique dual mechanism of action - the rapid, protein-reactive NHS-PEG, and the procoagulant collagen backing - serves as the advantage over other hemostatic pads. The use of a porous collagen matrix provides greater hemostatic effectiveness than oxidized cellulose, and a protein-binding layer adheres to the 
collagen pad more rapidly than a fibrinogen-thrombin-coated collagen pad. The versatility and utility of the hemostatic pad is replacing traditional hemostatic methods (eg, electrocautery, sutures) to improve surgical outcomes.

\section{Acknowledgments}

The authors would like to thank Woonza Rhee, Hans-Christian Hedrich, Joris Hoefinghoff, Johannes Regenbogen, Ed Osawa, Andreas Goppelt, Heinz Redl, Bernhard Baumgartner, Raymond Nistor, and the other numerous researchers and doctors who participated in the development of HEMOPATCH. The authors would also like to thank James P DiOrio and Mary Ann Murphy for their imaging expertise.

\section{Disclosure}

Drs KML, EK, and HG are employees of Baxter International Inc. subsidiaries. This review is based on peer-reviewed studies without modification of the cited authors' results or conclusions. The authors report no other conflicts of interest in this work.

\section{References}

1. Achneck HE, Sileshi B, Jamiolkowski RM, Albala DM, Shapiro ML, Lawson JH. A comprehensive review of topical hemostatic agents: efficacy and recommendations for use. Ann Surg. 2010;251(2): 217-228.

2. Silverstein E, Keown K, Chvapil M. Collagen fibers as a fleece hemostatic agent. J Trauma. 1980;20(8):688-694.

3. Schelling G, Block T, Blanke E, Hammer C, Brendel W, Gokel M. The effectiveness of a fibrinogen-thrombin-collagen-based hemostatic agent in an experimental arterial bleeding model. Ann Surg. 1987; 205(4):432-435.

4. Lewis KM, McKee J, Schiviz A, Bauer A, Wolfsegger M, Goppelt A. Randomized, controlled comparison of advanced hemostatic pads in hepatic surgical models. ISRN Surg. 2014;2014:930803.

5. Öllinger R, Mihaljevic AL, Schuhmacher C, et al. A multicentre, randomized clinical trial comparing the Veriset ${ }^{\mathrm{TM}}$ haemostatic patch with fibrin sealant for the management of bleeding during hepatic surgery. HPB (Oxford). 2013;15(7):548-558.

6. Fischer CP, Bochicchio G, Shen J, et al. A prospective, randomized controlled trial of the efficacy and safety of fibrin pad as an adjunct to control soft tissue bleeding during abdominal retroperitoneal, pelvic, and thoracic surgery. J Am Coll Surg. 2013;217(3):385-393.

7. The World Organization for Animal Health. The OIE Terrestrial Animal Health Code: Bovine Spongiform Encephalopathy. 2014;11.4. Available from: http:/www.oie.int/index.php?id=169\&L=0\&htmfile=chapitre bse.htm. Accessed June 16, 2015.

8. Fingerhut A, Uranues S, Ettorre GM, et al. European initial hands-on experience with HEMOPATCH, a novel sealing hemostatic patch: application in general, gastrointestinal, biliopancreatic, cardiac, and urologic surgery. Surg Technol Int. 2014;25:29-35.

9. Bouten PJM, Zonjee M, Bender J, et al. The chemistry of tissue adhesive materials. Prog Polmr Sci. 2014;39:1375-1405.

10. Borzelleca JF, Depukat K, Hallagan JB. Lifetime toxicity/carcinogenicity studies of FD and C Blue No 1 (brilliant blue FCF) in rats and mice. Food Chem Toxicol. 1990;28(4):221-234.

11. Flury M, Flühler H. Brilliant Blue FCF as dye tracer for solute transport studies - a toxicological review. J Environ Qual. 1994;23: 1108-1112.
12. Preul MC, Bichard WD, Spetzler RF. Toward optimal tissue sealants for neurosurgery: use of a novel hydrogel sealant in a canine durotomy repair model. Neurosurgery. 2003;53(5):1189-1198.

13. Mädler S, Bich C, Touboul D, Zenobi R. Chemical cross-linking with NHS esters: a systematic study on amino acid reactivities. J Mass Spectrom. 2009;44(5):694-706.

14. Allen MS, Wood DE, Hawkinson RW, et al. Prospective randomized study evaluating a biodegradable polymeric sealant for sealing intraoperative air leaks that occur during pulmonary resection. Ann Thorac Surg. 2004;77(5):1792-1801.

15. Lewis KM, Spazierer D, Slezak P, Baumgartner B, Regenbogen J, Gulle H. Swelling, sealing, and hemostatic ability of a novel biomaterial: a polyethylene glycol-coated collagen pad. J Biomater Appl. 2014;29(5): 780-788.

16. Gabay M. Absorbable hemostatic agents. Am J Health Syst Pharm. 2006;63(13):1244-1253.

17. Savage B, Saldivar E, Ruggeri ZM. Initiation of platelet adhesion by arrest onto fibrinogen or translocation on von Willebrand factor. Cell 1996;84(2):289-297.

18. Rand ML, Leung R, Packham MA. Platelet function assays. Transfus Apheresis Sci. 2003;28(3):307-317.

19. Hamad OA, Bäck J, Nilsson PH, et al. Platelets, complement, and contact activation: partners in inflammation and thrombosis. Adv Exp Med Biol. 2012;946:185-205.

20. Matonick JP, Hammond J. Hemostatic efficacy of EVARREST ${ }^{\mathrm{TM}}$, Fibrin Sealant Patch vs TachoSil ${ }^{\circledR}$ in a heparinized swine spleen incision model. J Invest Surg. 2014;27(6):360-365.

21. Wallace DG, Cruise GM, Rhee WM, et al. A tissue sealant based on reactive multifunctional polyethylene glycol. J Biomed Mater Res. 2001;58(5):545-555.

22. Lewis KM, Schiviz A, Hedrich HC, Regenbogen J, Goppelt A. Hemostatic efficacy of a novel, PEG-coated collagen pad in clinically relevant animal models. Int J Surg. 2014;12(9):940-944.

23. Mabry CD, Thompson BW, Read RC. Activated clotting time (ACT) monitoring of intraoperative heparinization in peripheral vascular surgery. Am J Surg. 1979;138(6):894-900.

24. Lewis KM, Atlee H, Mannone A, et al. Hemostatic effectiveness of two gelatin and thrombin combination hemostats. J Invest Surg. 2013; 26(3):141-148.

25. Lewis KM, Spazierer D, Urban M, Lin L, Goppelt A, Redl H. Comparison of regenerated and non-regenerated oxidized cellulose hemostatic agents. Eur Surg. 2013;45:213-220.

26. Rena O, Papalia E, Mineo TC, et al. Air-leak management after upper lobectomy in patients with fused fissure and chronic obstructive pulmonary disease: a pilot trial comparing sealant and standard treatment. Interact Cardiovasc Thorac Surg. 2009;9(6):973-977.

27. Marta GM, Facciolo F, Ladegaard L, et al. Efficacy and safety of TachoSil versus standard treatment of air leakage after pulmonary lobectomy. Eur J Cardiothorac Surg. 2010;38:683-689.

28. Imkamp F, Tolkach Y, Wolters M, Jutzi S, Kramer M, Herrmann T. Initial experiences with the Hemopatch ${ }^{\circledR}$ as a hemostatic agent in zero-ischemia partial nephrectomy. World J Urol. 2014;33(10): 1527-1534.

29. Prestipino F, Nenna A, Casacalenda A, Chello M. Ventricular perforation by pacemaker lead repaired with two hemostatic devices. Int J Surg Case Rep. 2014;5(12):906-908.

30. Jainandunsing JS, Al-Ansari S, Woltersom BD, Scheeren T, Natour E. Novel hemostatic patch achieves sutureless epicardial wound closure during complex cardiac surgery, a case report. J Cardiothorac Surg. 2015;10(1):12.

31. Navarro-Freire F, Navarro-Sanchez P, Garcia-Agua N, et al. Effectiveness of surgery in liver metastasis from colorectal cancer: experience and results of a continuous improvement process. Clin Transl Oncol. 2015;17(7):547-556.

32. Agrusa A, Romano G, Frazzetta G, et al. Laparoscopic adrenalectomy for large adrenal masses: single team experience. Int J Surg. 2014;12(1): S72-S74. 


\section{Publish your work in this journal}

Medical Devices: Evidence and Research is an international, peerreviewed, open access journal that focuses on the evidence, technology, research, and expert opinion supporting the use and application of medical devices in the diagnosis, treatment and management of clinical conditions and physiological processes. The identification of novel

devices and optimal use of existing devices which will lead to improved clinical outcomes and more effective patient management and safety is a key feature. The manuscript management system is completely online and includes a quick and fair peer-review system. Visit http://www. dovepress.com/testimonials.php to read real quotes from authors.

Submit your manuscript here: http://www.dovepress.com/medical-devices-evidence-and-research-journal 